\title{
Images in Cardiovascular Medicine \\ A Case of Successful Mitraclip for Severe Mitral Regurgitation with Left Ventricular Dysfunction in Korea
}

\section{OPEN ACCESS}

\section{Received: Feb 12, 2020}

Revised: Mar 6, 2020

Accepted: Mar 17, 2020

Correspondence to

Jung-Sun Kim, MD, PhD

Division of Cardiology, Severance

Cardiovascular Hospital, Yonsei University

College of Medicine, Yonsei-ro 50-1,

Seodaemun-gu, Seoul 03722, Korea.

E-mail: kjs1218@yuhs.ac

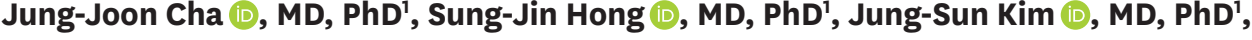
Jiwon Seo $\mathbb{D}$, MD', Seung Hyun Lee $\mathbb{D}, \mathrm{MD}, \mathrm{PhD}^{2}$, Sak Lee $\mathbb{1}, \mathbf{M D}, \mathrm{PhD}^{2}$, Chi Young Shim $\left(\mathbb{D}, \mathrm{MD}, \mathrm{PhD}^{\prime}\right.$, and Geu-Ru Hong $\mathbb{D}^{-}, \mathrm{MD}, \mathrm{PhD}$

'Division of Cardiology, Severance Cardiovascular Hospital, Yonsei University College of Medicine, Yonsei University Health System, Seoul, Korea

${ }^{2}$ Division of Cardiovascular Surgery, Severance Cardiovascular Hospital, Yonsei University College of Medicine, Yonsei University Health System, Seoul, Korea

The patient was an 81-year-old female with medical treatment for old myocardial infarction which occurred in the middle left anterior descending artery in 1989. She had multiple admission for heart failure, with the most recent admission being associated with aggravated symptoms by low left ventricular (LV) ejection fraction with severe tricuspid regurgitation (TR) despite optimal medical therapy. Chest X-ray showed cardiomegaly with bilateral pleural effusion. As shown in Figures 1 and 2, echocardiography demonstrated a
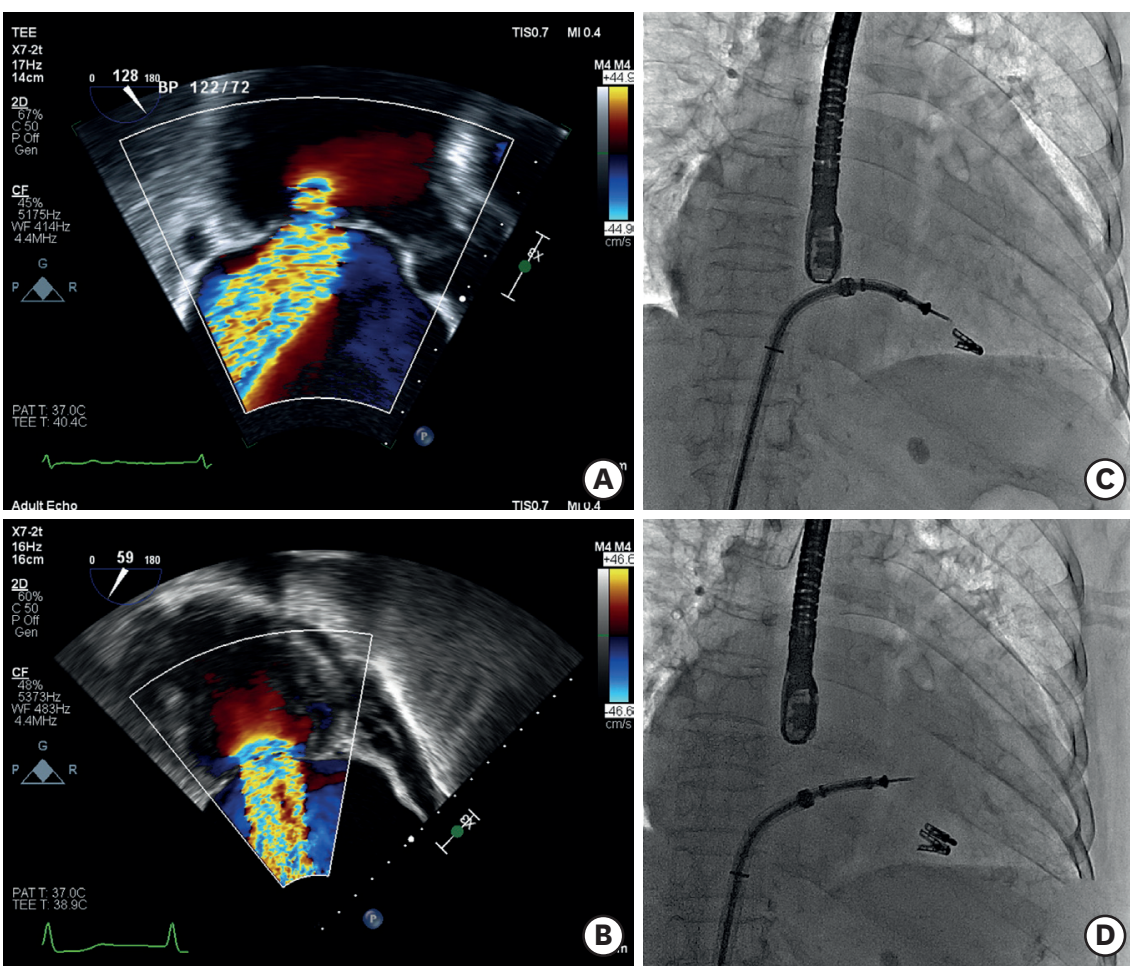
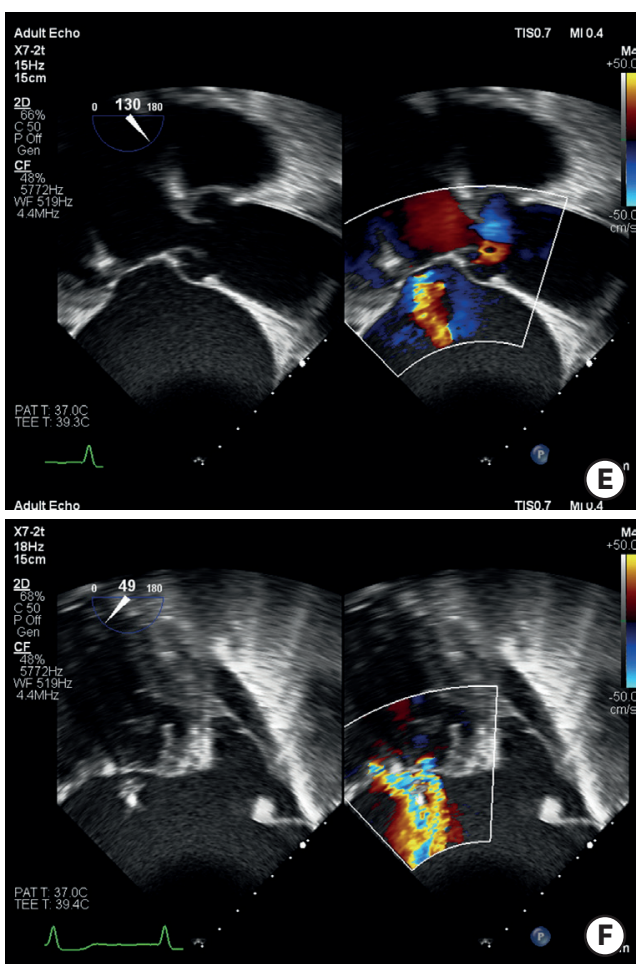

Figure 1. Severe MR demonstrated on (A, B) Pre-procedural transesophageal echocardiographic left ventricualar outflow tract and intercommissural view. (C) First grasp. (D) Second grasp. Reduced MR reduced after clipping demonstrated on (E,F) Post-procedural transesophageal echocardiographic left ventricualar outflow tract and intercommissural view. $\mathrm{MR}=$ mitral regurgitation . 
Copyright (C) 2020. The Korean Society of Cardiology

This is an Open Access article distributed under the terms of the Creative Commons Attribution Non-Commercial License (https:// creativecommons.org/licenses/by-nc/4.0) which permits unrestricted noncommercial use, distribution, and reproduction in any medium, provided the original work is properly cited.

\section{ORCID iDs}

Jung-Joon Cha (iD)

https://orcid.org/0000-0002-8299-1877 Sung-Jin Hong (iD

https://orcid.org/0000-0003-4893-039X

Jung-Sun Kim iD

https://orcid.org/0000-0003-2263-3274 degenerative mitral valve with a prolaptic motion of anterior mitral leaflet with tethering (Supplementary Videos 1 and 2). There was incomplete coaptation of A2-P2. This resulted in severe mitral regurgitation (MR) with an effective regurgitant orifice area (EROA) of $0.68 \mathrm{~cm}^{2}$, the regurgitant volume of $73 \mathrm{~mL}$. She had enlarged LV dimensions and LV ejection fraction of $42 \%$ due to old myocardial infarction. The Society of Thoracic Surgeons score for mortality was $12.3 \%$. After a Heart Team discussion, the patient was offered the transcatheter mitral-valve repair according to recent European Society of Cardiology guidelines (Class IIb). ${ }^{1-3)}$ The first grasp was performed centrally with careful attention to grasp both leaflets (A2-P2) adequately. With a single clip, the MR was reduced from IV to III. Thus, the second grasp was performed at the lateral side parallel to the first clip. After the second grasp (Supplementary Videos 3), MR was moderate in severity with EROA of 0.21 $\mathrm{cm}^{2}$ and regurgitant volume of $36 \mathrm{~mL}$ (Supplementary Videos $\mathbf{4}$ and 5). Additionally, TR was reduced from IV to III. She was discharged with improved functional status from New York Heart Association Classification IV to II.
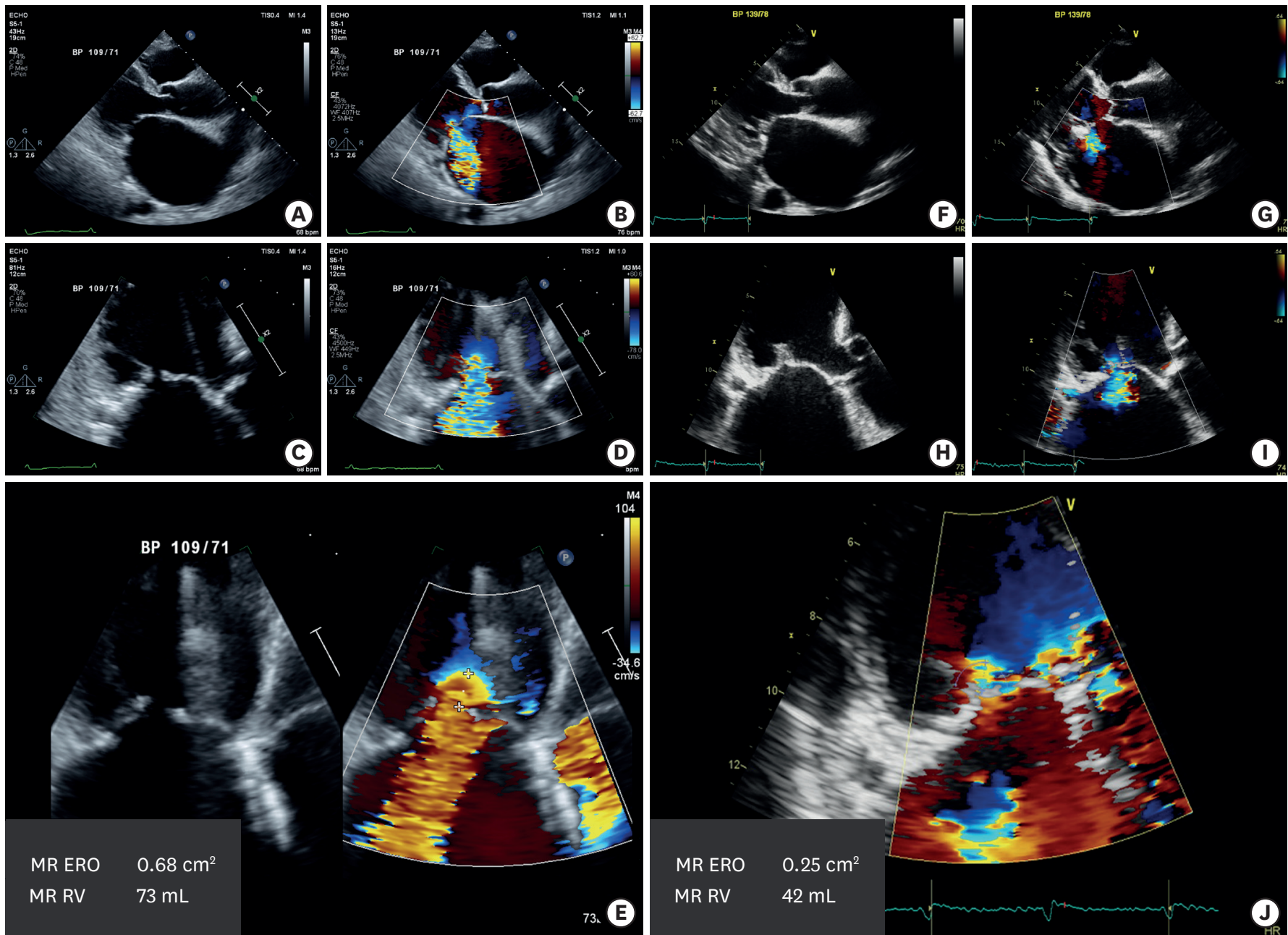

Figure 2. (A, B) Transthoracic echocardiographic parasternal long axis and (C,D) apical 3-chamber views showing degenerative mitral valve with severe MR. (E) pre-procedural EROA and regurgitant volume is $0.68 \mathrm{~cm}^{2}$ and $73 \mathrm{~mL}$, respectively. After the MitraClip, (F, G) transthoracic echocardiographic parasternal long axis and $(\mathrm{H}, \mathrm{I})$ apical 3-chamber views showing degenerative mitral valve with severe $\mathrm{MR}$. $(\mathrm{J})$ post-procedural EROA and regurgitant volume is $0.21 \mathrm{~cm}^{2}$ and 36 $\mathrm{mL}$, respectively.

$\mathrm{MR}=$ mitral regurgitation, $\mathrm{EROA}=$ effective regurgitant orifice area 


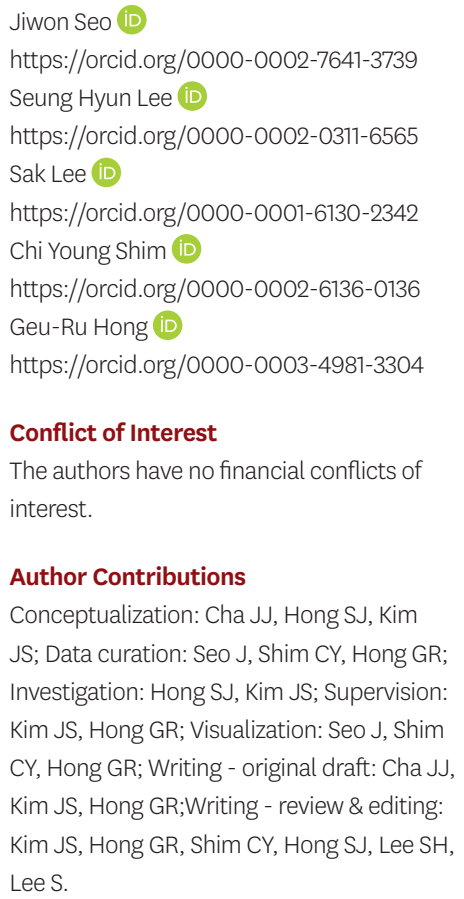

\section{SUPPLEMENTARY MATERIALS}

\section{Supplementary Video 1}

Transthoracic echocardiography demonstrated severe mitral regurgitation with a prolaptic motion of anterior mitral leaflet.

Click here to view

\section{Supplementary Video 2}

Transesophageal echocardiography showed severe mitral regurgitation due to incomplete coaptation of A2-P2.

Click here to view

\section{Supplementary Video 3}

Successful MitraClip was performed with two clips.

Click here to view

\section{Supplementary Video 4}

Transthoracic echocardiography demonstrated mitral regurgitation was reduced from IV to II.

Click here to view

\section{Supplementary Video 5}

Transesophageal echocardiography showed the clips were well positioned with reduced mitral regurgitation.

Click here to view

\section{REFERENCES}

1. Baumgartner H, Falk V, Bax JJ, et al. 2017 ESC/EACTS Guidelines for the management of valvular heart disease. Eur Heart J 2017;38:2739-91.

PUBMED | CROSSREF

2. Stone GW, Lindenfeld J, Abraham WT, et al. Transcatheter mitral-valve repair in patients with heart failure. N Engl J Med 2018;379:2307-18.

PUBMED | CROSSREF

3. Choi JY, Hong GR. Transcatheter mitral valve repair: growing evidence regarding it's efficacy and optimal indication. Korean Circ J 2019;49:542-4.

PUBMED | CROSSREF 